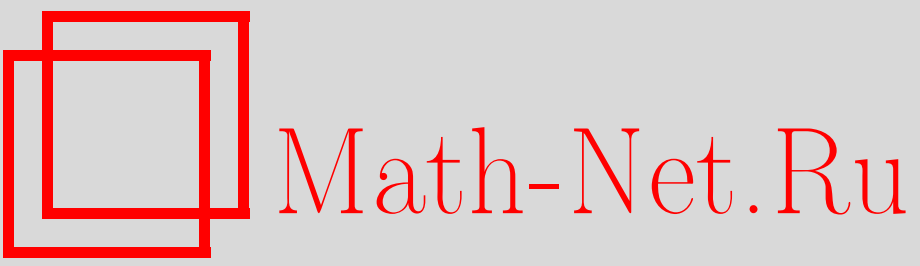

К. Э. Фельдман, Роды Хирцебруха многообразий, несущих гамильтоново действие окружности, УМH, 2001, том 56, выпуск 5, 187-188

DOI: https://doi.org/10.4213/rm446

Использование Общероссийского математического портала Math-Net.Ru подразумевает, что вы прочитали и согласны с пользовательским соглашением

http://www.mathnet.ru/rus/agreement

Параметры загрузки:

IP: 54.197 .130 .99

26 апреля 2023 г., 14:59:26 


\title{
РОДЫ ХИРЦЕБРУХА МНОГООБРАЗИЙ, НЕСУЩИХ ГАМИЛЬТОНОВО ДЕЙСТВИЕ ОКРУЖНОСТИ
}

\author{
К.Э. ФЕЛЬДМАН
}

В заметке описьваются препятствия к существованию симплектических и гамильтоновых симплектических действий окружности на замкнутых компактных многообразиях в терминах родов Хирцебруха и соотношения между дифференциальными и гомотопическими инвариантами таких многообразий. Все рассматриваемые многообразия предполагаются замкнутыми и компактными.

Теорема 1. Род Тодда многообразия, допускающего симплектическое действие окружности с изолированными неподвижными точками, равен либо нулю, и тогда действие негамильтоново; либо единице, и тогда действие гамильтоново. Любое симплектическое действие окружности на многообразии с положительным родом Тодда всегда гамильтоново.

ДокАЗАТЕЛЬСтво. Симплектическое действие окружности гамильтоново тогда и только тогда, когда существует связная компонента неподвижных точек, все веса представления окружности в нормальном расслоении которой положительны. Эта компонента связности единственна и соответствует глобальному минимуму отображения моментов (см. подробности в [1]). Воспользуемся формулой о неподвижных точках для рода Хирцебруха $\chi y$, соответствующего степенному ряду $\frac{x\left(1+y e^{-x(1+y)}\right)}{1-e^{-x(1+y)}}$. Пусть $M_{s}$ - связные компоненты множества неподвижных точек действия окружности, $d_{s}$ - число отрицательных весов представления окружности в нормальном расслоении к $M_{s}$, тогда (см. [2])

$$
\chi_{y}(M)=\sum_{s}(-y)^{d_{s}} \chi_{y}\left(M_{s}\right),
$$

где суммирование ведется по всем компонентам связности множества неподвижных точек. Поскольку $\chi_{y}(p t)=1$, свободньй член рода $\chi y$ многообразия, оснащенного гамильтоновьм действием окружности с изолированными неподвижньми точками, равен единице. Наоборот, если свободный член в $\chi_{y}(M)$ отличен от нуля, то любое симплектическое действие окружности на $M$ гамильтоново. Утверждение теоремы вытекает из $T d(M)=\left.\chi_{y}(M)\right|_{y=0}$.

Резултат теоремы 1 является частньм случаем ограничений, налагаемых уравнениями Коннера-Флойда на многообразия с действием окружности. Зафиксируем мультипликативный род $h: \Omega_{*}^{U} \rightarrow Q$. Пусть $g_{h}(u)=u+\sum \frac{h([C P(n)])}{n+1} u^{n+1}$. Для почти комплексного многообразия $M^{2 n}$, оснащенного согласованным действием окружности с изолированными неподвижными точками, сумма по всем неподвижным точкам произведений $\prod_{k=1}^{n}\left(g_{h}^{-1}\left(j_{k} g_{h}(u)\right)\right)^{-1}$ $\left(j_{k}, k=1, \ldots, n,-\right.$ веса представления окружности в окрестности рассматриваемой неподвижной точки) не содержит отрицательных степеней по $u$, а свободный член равен $h\left(M^{2 n}\right)$ [3], [4].

СледСТВИЕ 1 [5]. Полусвободное симплектическое действие окружности с изолированными неподвижными точками гамильтоново тогда и только тогда, когда множество неподвижных точек непусто.

ДокАЗАТЕЛЬСтво. Выпишем уравнения Коннера-Флойда для рода Тодда в этой ситуации. Поскольку $T d([C P(m)])=1$ для всех $m$, имеем $g_{T d}(u)=\sum u^{m+1} /(m+1)=\ln (1-u)$, $g_{T d}^{-1}\left(j g_{T d}(u)\right)=1-(1-u)^{j}$. Все веса представлений окружности для полусвободного действия равны \pm 1 . Суммируя по всем неподвижным точкам, получаем, что функция $f(u)=$ $\sum_{k=1}^{2 n} F_{k}(u-1)^{k} / u^{n}$, где $F_{k}$ - число неподвижных точек, содержащих ровно $k$ отрицательных

Работа выполнена при поддержке стипендии Сегги Брауна университета г. Эдинбурга. 
весов, является некоторой константой $\lambda$, равной роду Тодда многообразия. Решая соответствующую систему линейных по $F_{k}$ уравнений, находим $\left(F_{0}, F_{1}, \ldots, F_{n}\right)=\lambda\left(\left(\begin{array}{c}n \\ 0\end{array}\right),\left(\begin{array}{c}n \\ 1\end{array}\right), \ldots,\left(\begin{array}{c}n \\ n\end{array}\right)\right)$. Применяя теорему 1, получаем утверждение следствия.

СледСтвиЕ 2 [6]. Симплектическое действие окружности на 4-мерном многообразии гамильтоново тогда и только тогда, когда множество неподвижных точек неnусто.

ДокАЗАТЕльСтво. Покажем, что наличие неподвижных точек влечет существование неподвижной точки, в окрестности которой веса представления окружности имеют один и тот же знак, и, следовательно, такая точка является максимумом или минимумом отображения моментов. Предположим противное. Тогда не существует компонент связности неподвижных точек коразмерности два (такая компонента имеет ровно один вес). Значит, все неподвижные точки изолированы и содержат в точности один положительный и один отрицательный веса. Противоречие следует из следующего утверждения.

ПрЕДЛОЖЕНИЕ. Почти комплексное действие окружности с изолированными неподвижными точками на $M^{2 n}$ всегда имеет неподвижные точки, числа отричательных весов представлений окружности в нормальных расслоениях которых имеют разную четность.

ДОКАЗАТЕЛЬСТВО. В противном случае коэффициенты при $1 / u^{n}$ в уравнениях КоннераФлойда для рода Тодда этого многообразия имеют один и тот же знак.

Рассмотрим более детально случай гамильтонова действия окружности. Обозначим через $P_{M}(t)$ полином Пуанкаре многообразия $M$.

Теорема 2. Если симплектическое многообразие $M$ допускает гамильтоново действие окружности с изолированными неподвижными точками, то $\chi_{y}(M)=P_{M}(\sqrt{-y})$.

ДоКАЗАТЕльство. Критические точки отображения моментов совпадают с неподвижными точками действия окружности. Индекс каждой критической точки равен удвоенному числу отрицательных весов представления окружности. Утверждение вытекает из (1).

СледСтвИЕ 3 [7]. Сигнатура и числа Бетти многообразия $M^{4 n}$, допускающего гамильтоново действие окружности с изолированными неподвижными точками, связаны соотношением: $\sigma\left(M^{4 n}\right)=\sum_{k=0}^{n} b_{4 k}-\sum_{k=0}^{n-1} b_{4 k+2}$.

ДоКАЗАТЕЛЬСТво. Утверждение следует из теоремы 2 после подстановки $y=1$.

СледСтВИЕ 4. У гомотопически әквивалентных многообразий, допускающих гамильтоново действие окружности с изолированными неподвижными точками, ұу-родь равныл.

ЗАмЕчАНИЕ 1. Аналогично [8] для негамильтонова симплектического действия окружности с изолированными неподвижными точками $\chi_{y}\left(M^{2 n}\right)=\sum_{k=0}^{n} b_{2 k}(-y)^{k}$, где $b_{k}$ - числа Новикова [9], соответствующие обобщенному отображению моментов. Было бы важно построить пример такого действия с непустым множеством неподвижных точек.

\section{СПИСОК ЛИТЕРАТУРЫ}

[1] M. Audin. The Topology of Torus Actions on Symplectic Manifolds. Basel: Birkhäuser, 1991. (Progr. Math. V. 93.) [2] F. Hirzebruch, T. Berger, R. Jung. Manifolds and Modular Forms. Braunschweig: Vieweg, 1992. [3] С.П. Новиков // Изв. АН СССР. Сер. матем. 1968. Т. 32. С. 1245-1263. [4] И. М. Кричевер // Изв. АН СССР. Сер. матем. 1974. Т. 38. C. 1289-1304. [5] S. Tolman, J. Weitsman // Topology. 2000. V. 39. № 2. P. 299-309. [6] D. McDuff // J. Geom. Phys. 1988. V. 5. № 2. P. 149-160. [7] J. D. S. Jones, J. H. Rawnsley // J. Geom. Phys. 1997. V. 23. № 3-4. P. 301-307. [8] M. Фарбер // УМН. 1999. T. 54. № 1. С. 209-212. [9] С. П. Новиков // УМН. 1982. Т. 37. № 5. С. 3-49.

University of Edinburgh

E-mail: feldman@maths.ed.ac.uk
Принято редколлегией 22.08 .2001 